\title{
Does Pre-Operative Tranexamic Acid Increase the Incidence of Thromboembolism in Primary Lower Limb Arthroplasty?
}

\author{
Veenesh Selvaratnam*, James R. Fountain, Nigel J. Donnachie, Thomas G. Thomas, Fintan A. Carroll \\ Wirral University Teaching Hospital NHS Foundation Trust, Wirral, UK. \\ Email: *veenesh_selvaratnam@yahoo.co.uk
}

Received July $11^{\text {th }}$, 2013; revised August $15^{\text {th }}$, 2013; accepted September $1^{\text {st }}, 2013$

Copyright $(\odot 2013$ Veenesh Selvaratnam et al. This is an open access article distributed under the Creative Commons Attribution License, which permits unrestricted use, distribution, and reproduction in any medium, provided the original work is properly cited.

\begin{abstract}
Introduction: Tranexamic Acid (TA) has been shown to decrease peri-operative bleeding in primary lower limb arthroplasty surgery. There are still concerns with regards to the increased risk of thromboembolic events with the use of TA. The aim of this study was to assess whether the use of pre-operative TA increased the incidence of Deep Vein Thrombosis (DVT) and Pulmonary Embolism (PE) in Total Hip Replacement (THR) and Total Knee Replacement (TKR). Methods: Patients who underwent primary THR or TKR between August 2007 and August 2009 were identified from the databases of three surgeons within the lower limb arthroplasty unit. A retrospective case notes analysis was performed. DVT was diagnosed on Duplex Ultrasound Scan and PE on CT Pulmonary Angiogram. A positive result was a diagnosis of DVT or PE within 3 months of surgery. Results: 701 patients underwent primary THR and TKR over the 2-year period. 320 patients (189 THR, 131 TKR) received TA pre-operatively. 381 patients (190 THR, 191 TKR) did not receive TA prior to surgery. A total of 8 (2.5\%) patients who received TA were diagnosed with either a DVT (4) or PE (4) post operatively. In those patients not receiving TA, 6 had a DVT and 4 had a PE, a total of 10 (2.6\%). Conclusion: Pre-operative use of Tranexamic Acid in primary THR and TKR does not increase the incidence of DVT and PE.
\end{abstract}

Keywords: Tranexamic Acid; DVT; PE; TKR; THR

\section{Introduction}

Post operative anaemia following lower limb arthroplasty delays rehabilitation and discharge with the increased complications and costs associated with an extended inpatient stay. A variety of blood conserving techniques have been developed to reduce blood loss and post-operative transfusion rates. These include autologous blood transfusion, intra-operative blood salvage, controlled hypotension and the use of erythropoietin and antifibrinolytic agents.

Antifibrinolytics currently include aprotinin and tranexamic acid (TA). TA is an analogue of the amino acid lysine. It inhibits fibrinolysis by blocking the lysine binding sites of plasminogen $[1,2]$ reducing the conversion of plasminogen to plasmin. The enzyme plasmin is responsible for the degradation of fibrin clot, fibrinogen and other plasma proteins including the procoagulant factors $\mathrm{V}$ and VIII. Together these form the basic framework for

${ }^{*}$ Corresponding author. the formation of a blood clot in heamostasis. At higher doses, TA can directly inhibit plasmin rather than preventing its formation.

TA has been used in urological, gynaecological and cardiothoracic surgery to reduce blood loss and has been shown to decrease peri-operative bleeding in primary lower limb arthroplasty surgery [2-9]. There are still concerns with regards to the increased risk of thromboembolic events with the use of TA in the clinical setting. Although TA may dramatically reduce blood loss [10] it has yet to gain widespread acceptance amongst those performing lower limb arthroplasties. The aim of this study was to assess whether the use of pre-operative TA increased the incidence of Deep Vein Thrombosis (DVT) and Pulmonary Embolism (PE) in Total Hip Replacement (THR) and Total Knee Replacement (TKR).

\section{Materials and Methods}

The local audit department approval was obtained. Pa- 
tients who underwent primary THR or TKR between August 2007 and August 2009 were identified from the databases of three surgeons within the lower limb arthroplasty unit.

The criteria for inclusion in this study were:

1) patients who underwent primary TKR or THR

2) a diagnosis of osteoarthritis, rheumatoid arthritis and aseptic bone necrosis

3) no prior history of venous thromboembolic (VTE) disease

4) no history of bleeding disorder

5) not on any prior anticoagulant

A retrospective case notes analysis was performed comparing patients who received TA and those who did not receive TA during TKR and THR.

The dose of TA used was a single bolus dose of 10 $\mathrm{mg} / \mathrm{kg}$ body weight given intravenously 30 minutes prior to anaesthetic induction. TA given intravenously diffuses rapidly into synovial fluid and membrane [11] and maximum plasma levels are reached in 5 to 15 minutes. The haemostatic effect of TA has been shown to be most effective when administered prior to the operation allowing suppression of fibrinolysis from the start of surgery [10].

All patients had the same pre and peri-operative care and were followed up post operatively at 6 weeks, 6 months and then yearly.

In accordance with the NICE guidelines, all our patients who underwent THR had the appropriate chemical VTE prophylaxis during their inpatient stay. Full leg graduated compression elastic stockings were worn postoperatively by all patients until fully mobile. Chemical VTE prophylaxis was started six hours after surgical closure in all patients provided haemostasis had been established. At the time all patients received thromboprophylaxis with a low molecular weight heparin for 14 days and 28 days post operatively for TKR and THR respectively.

Following clinical examination of a symptomatic patient, DVT was diagnosed on duplex ultrasound scan and PE on CT pulmonary angiography (CTPA). A positive result was a diagnosis of DVT or PE within 3 months of surgery.

\section{Results}

A total of 701 patients underwent lower limb arthroplasty over the two year period, with 379 patients having primary THR and 322 patients receiving a TKR.

The 379 patients undergoing primary THR had an age range from 54 - 88 years (median: 72 years). 189 patients received TA pre-operatively. 190 patients did not receive TA prior to surgery. A total of 4 (2.1\%) patients who received TA were diagnosed with either a DVT (2) or PE (2) post operatively. One patient had a below knee DVT while the other had an above knee DVT. In those patients not receiving TA, 2 patients had a PE (1.1\%). This is statistically not significant $\mathrm{p}=0.184$.

322 patients underwent primary TKR over the 2 year period. Their age range was from 55 - 90 years (median: 74 years). 131 patients received TA pre-operatively. 191 patients did not receive TA prior to surgery. A total of 4 (3.1\%) patients who received TA were diagnosed with a thromboembolic event post-operatively. 2 with DVT and 2 with a PE. Both patient had a below knee DVT. In those patients not receiving TA, 6 had a DVT and 2 had a PE, a total of 8 (4.2\%). Three had an above knee DVT while the other three had an above knee DVT. Patients of both groups with post operative venous thromboembolic (VTE) disease were anticoagulated with warfarin (3 months for DVT and 6 months for PE).

None of the patients in our study died as a direct result of VTE and there were no adverse reactions to TA in all patients.

\section{Discussion}

A higher haemoglobin following lower limb arthroplasty has been associated with a significantly shorter length of stay for inpatient rehabilitation and improved patient function score [12].

VTE is a relatively common, occasionally fatal and costly complication of elective TKR and THR surgery. The reported prevalence of DVT in patients not receiving prophylaxis in clinical trials using mandatory venography has been $45 \%$ - 57\% after THR [13] and 40\% - 84\% after TKR [14]. The reported prevalence of PE in other trials has been $0.7 \%$ - 30\% [15] after THR (fatal PE 0.1\% - 0.4\%), and 1.8\% - 7\% after TKR (fatal PE 0.2\% - 0.7\%) [16].

This risk of VTE in lower limb arthroplasty surgery is theoretically increased with the use of antifibrinolytic agents such as TA but it would seem the effect of TA is more pronounced in operative wounds than in the peripheral venous blood [4]. The reduced thrombogenic effect in the peripheral venous system is potentially because the fibrinolytic activity in vein walls is not affected by TA.

The therapeutic level of TA is between $5-10 \mathrm{mg} / \mathrm{l}$ [17]. A pre-operative IV dose of $10 \mathrm{mg} / \mathrm{kg}$ of TA gives a plasma level of approximately $10 \mathrm{mg} / \mathrm{l}$ and therefore a therapeutic concentration for the period of hyperfibrinolysis associated with the peri-operative period of arthroplasty surgery [7]. TA was first introduced in arthroplasty surgery in order to reduce peri-operative blood loss and it has been proven to do that effectively. Post-operative blood loss is maximal in the first eight hours [18] and it has been shown subsequently that a single dose of TA is enough to cover this immediate post-operative period [19]. Other authors would argue the need for further 
post operative doses but with a therapeutic plasma concentration of $5 \mathrm{mg} / \mathrm{l}$ still present at 18 hours after a dose of $10 \mathrm{mg} / \mathrm{kg}$ [17] we feel a single dose is adequate.

A single pre-operative bolus dose at induction has been used in other studies [8] while other trials have combined a pre-operative dose with intra-operative infusions or further post operative doses $[7,20]$.

Ralley used one $20 \mathrm{mg} / \mathrm{kg}$ dose of TA before skin incision for THA or 10 minutes before tourniquet release in TKA [8] while Orpen used $15 \mathrm{mg} / \mathrm{kg}$ of TA given IV at the start of cement mixing in TKA [7].

Sukeik et al. [21] analysed 11 randomised controlled trials with pre-operative doses that ranged from $10 \mathrm{mg} / \mathrm{kg}$ to $30 \mathrm{mg} / \mathrm{kg}$, the biggest proportion only using a single bolus pre-operatively.

The use of LMWH thromboprophylaxis for all our patients follows NICE guidance and is in keeping with Sudeik's [21] meta-analysis in which the majority of trials used LMWH as chemical DVT prophylaxis.

Only patients who had a clinical DVT on examination were investigated with a duplex ultrasound Scan. Clinically suspected PE was investigated with CT Pulmonary Angiography (CTPA). Duplex ultrasound scan has a sensitivity of $97 \%$ and a specificity of $98 \%$ for the diagnosis of DVT [22] while CTPA remains the gold standard for the diagnosis of PE [23].

Our results have confirmed the VTE findings in other published data. Tanaka et al. found no difference in DVTs between a group receiving TA and their control [10]. Lozano et al. [9] studying the effectiveness and safety of TA reported on 414 pts who had been examined clinically and by contrast venography. They showed a lower frequency of clinically evident thromboembolic complications in the cohort treated with TA compared to those without.

Sudeik et al. found no significant differences in DVT or PE among the randomized controlled trials included in the meta-analysis. All studies had patients in the intervention group receiving IV TA and a control group receiving placebo, another antifibrinolytic or no treatment [21]. 9 out of the 11 trials used clinical evaluation as a screen for DVT in symptomatic post operative patients in keeping with our protocols for further investigation.

Orpen et al. used duplex ultrasound as an assessment of the proximal deep venous system for DVT on all patients five days post TKA in their prospective trial of TA. No patient developed clinical signs or symptoms of DVT and there was no evidence of thromboembolism on duplex performed [7].

Benoni had 4 (9.3\%) confirmed DVTs in 43 TKR patients treated with TA compared with 3 (7\%) DVTs and $1 \mathrm{PE}$ in their placebo group of the same number [2]. Although a higher percentage of VTE events the difference between the prophylactic and placebo groups remains nonsignificant.

\section{Conclusion}

In summary, our report shows that the use of pre-operative tranexamic acid in primary total knee and hip replacement does not increase the incidence of DVT and PE. TA can be integrated easily into routine practice and it would appear that the beneficial effects of reduced blood loss, reduced transfusion requirements and increased haemoglobin levels at discharge are not accompanied with an increase in thromboembolic complications.

\section{REFERENCES}

[1] I. M. Nilsson, "Clinical Pharmacology of Aminocaproic and Tranexamic Acids,” Journal of Clinical Pathology, Vol. 14, No. 33, 1980, pp. 41-47.

[2] G. Benoni and H. Fredin, "Fibrinolytic Inhibition with Tranexamic Acid Reduces Blood Loss and Blood Transfusion after Knee Arthroplasty: A Prospective, Randomised, Double-Blind Study of 86 Patients," The Journal of Bone \& Joint Surgery, Vol. 78, No. 3, 1996, pp. 434-440.

[3] K. M. Ho and H. Ismail, "Use of Intravenous Tranexamic Acid to Reduce Allogeneic Blood Transfusion in Total Hip and Knee Arthroplasty: A Meta-Analysis,” Anaesthesia and Intensive Care, Vol. 31, No. 5, 2003, pp. 529537.

[4] G. Benoni, S. Lethagen and H. Fredin, "The Effect of Tranexamic Acid on Local and Plasma Fibrinolysis during Total Knee Arthroplasty,” Thrombosis Research, Vol. 85, No. 3, 1997, pp. 195-206. http://dx.doi.org/10.1016/S0049-3848(97)00004-2

[5] M. A. Camarasa, G. Olle, M. Serra-Prat, et al., "Efficacy of Aminocaproic, Tranexamic Acids in the Control of Bleeding during Total Knee Replacement: A Randomized Clinical Trial,” British Journal of Anaesthesia, Vol. 96, No. 5, 2006, pp. 576-582. http://dx.doi.org/10.1093/bja/ael057

[6] J. C. Alvarez, F. X. Santiveri, I. Ramos, E. Vela, L. Puig and F. Escolano, "Tranexamic Acid Reduces Blood Transfusion in Total Knee Arthroplasty Even When a Blood Conservation Program Is Applied,” Transfusion, Vol. 48, No. 3, 2008, pp. 519-525. http://dx.doi.org/10.1111/j.1537-2995.2007.01564.x

[7] N. M. Orpen, C. Little, G. Walker and E. J. Crawfurd, "Tranexamic Acid Reduces Early Post-Operative Blood Loss after Total Knee Arthroplasty: A Prospective Randomised Controlled Trial of 29 Patients,” The Knee, Vol. 13, No. 2, 2006, pp. 106-110. http://dx.doi.org/10.1016/j.knee.2005.11.001

[8] F. E. Ralley, D. Berta, V. Binns, J. Howard and D. D. Naudie, "One Intraoperative Dose of Tranexamic Acid for Patients Having Primary Hip or Knee Arthroplasty,” Clinical Orthopaedics and Related Research, Vol. 468, No. 7, 2010, pp. 1905-1911.

http://dx.doi.org/10.1007/s11999-009-1217-8 
[9] M. Lozano, M. Basora, L. Peidro, et al., "Effectiveness and Safety of Tranexamic Acid Administration during Total Knee Arthroplasty,” Vox Sanguinis, Vol. 95, No. 1, 2008, pp. 39-44. http://dx.doi.org/10.1111/j.1423-0410.2008.01045.x

[10] N. Tanaka, H. Sakahashi, E. Sato, K. Hirose, T. Ishima and S. Ishii, "Timing of the Administration of Tranexamic Acid for Maximum Reduction in Blood Loss in Arthroplasty of the Knee," The Journal of Bone \& Joint Surgery, Vol. 83, No. 5, 2001, pp. 702-705. http://dx.doi.org/10.1302/0301-620X.83B5.11745

[11] A. Ahlberg, O. Eriksson and H. Kjellman, "Diffusion of Tranexamic Acid to the Joint,” Acta orthopaedica Scandinavica, Vol. 47, No. 5, 1976, pp. 486-488. http://dx.doi.org/10.3109/17453677608988725

[12] P. T. Diamond, M. R. Conaway, S. H. Mody and K. Bhirangi, "Influence of Hemoglobin Levels on Inpatient Rehabilitation Outcomes after Total Knee Arthroplasty,” The Journal of Arthroplasty, Vol. 21, No. 5, 2006, pp. 636641. http://dx.doi.org/10.1016/j.arth.2005.09.006

[13] A. G. Turpie, M. N. Levine, J. Hirsh, et al., “A Randomized Controlled Trial of a Low-Molecular-Weight Heparin (Enoxaparin) to Prevent Deep-Vein Thrombosis in Patients Undergoing Elective Hip Surgery,” The New England Journal of Medicine, Vol. 315, No. 15, 1986, pp. 925-929. http://dx.doi.org/10.1056/NEJM198610093151503

[14] S. H. Cohen, G. E. Ehrlich, M. S. Kauffman and C. Cope, “Thrombophlebitis Following Knee Surgery," The Journal of Bone and Joint Surgery, Vol. 55, No. 1, 1973, pp. 106-112.

[15] B. I. Eriksson, P. Kalebo, B. A. Anthymyr, H. Wadenvik, L. Tengborn and B. Risberg, "Prevention of Deep-Vein Thrombosis and Pulmonary Embolism after Total Hip Replacement. Comparison of Low-Molecular-Weight Heparin and Unfractionated Heparin," The Journal of Bone and Joint Surgery, Vol. 73, No. 4, 1991, pp. 484-93.

[16] P. S. Wells, A. W. Lensing, B. L. Davidson, M. H. Prins and J. Hirsh, "Accuracy of Ultrasound for the Diagnosis of Deep Venous Thrombosis in Asymptomatic Patients after Orthopedic Surgery. A Meta-Analysis,” Annals of Internal Medicine, Vol. 122, No. 1, 1995, pp. 47-53. http://dx.doi.org/10.7326/0003-4819-122-1-199501010-0 $\underline{0008}$

[17] C. Dollery, “Therapeutic Drugs,” 2nd Edition, Churchill Livingstone, Edinburgh, 1998.

[18] G. Senthil Kumar, O. A. Von Arx and J. L. Pozo, "Rate of Blood Loss over 48 Hours Following Total Knee Replacement,” The Knee, Vol. 12, No. 4, 2005, pp. 307-309. http://dx.doi.org/10.1016/j.knee.2004.08.008

[19] L. McGonagle and S. Hakkalamani, "FAC Tranexamic Acid and Re-Infusion Drains in Total Knee Arthroplasty," European Journal of Orthopaedic Surgery \& Traumatology, Vol. 20, No. 7, 2010, pp. 553-555. http://dx.doi.org/10.1007/s00590-010-0625-5

[20] D. O. Molloy, H. A. Archbold, L. Ogonda, J. McConway, R. K. Wilson and D. E. Beverland, "Comparison of Topical Fibrin Spray and Tranexamic Acid on Blood Loss after Total Knee Replacement: A Prospective, Randomised Controlled Trial," The Journal of Bone \& Joint Surgery, Vol. 89, No. 3, 2007, pp. 306-309. http://dx.doi.org/10.1302/0301-620X.89B3.17565

[21] M. Sukeik, S. Alshryda, F. S. Haddad and J. M. Mason, "Systematic Review and Meta-Analysis of the Use of Tranexamic Acid in Total Hip Replacement," The Journal of Bone \& Joint Surgery, Vol. 93, No. 1, 2011, pp. 39-46. http://dx.doi.org/10.1302/0301-620X.93B1.24984

[22] R. Quintavalla, P. Larini, A. Miselli, et al., “Duplex Ultrasound Diagnosis of Symptomatic Proximal Deep Vein Thrombosis of Lower Limbs,” European Journal of Radiology, Vol. 15, No. 1, 1992, pp. 32-36. http://dx.doi.org/10.1016/0720-048X(92)90199-J

[23] Y. M. R. M. Estrada and S. A. Oldham, “CTPA as the Gold Standard for the Diagnosis of Pulmonary Embolism. International Journal of Computer Assisted Radiology and Surgery, Vol. 6, No. 4, 2010, pp. 557-563. http://dx.doi.org/10.1007/s11548-010-0526-4 